\title{
The molecular biology of diffuse large B-cell lymphoma
}

\author{
Mareike Frick, Bernd Dörken and Georg Lenz
}

\begin{abstract}
Diffuse large B-cell lymphoma (DLBCL) represents the most common type of malignant lymphoma. In the last few years, significant progress has been achieved in the understanding of the molecular pathogenesis of this entity. Gene expression profiling has identified three molecular DLBCL subtypes, termed germinal-center B-cell-like (GCB) DLBCL, activated $B$-cell-like $(A B C) D L B C L$, and primary mediastinal $B$-cell lymphoma (PMBL). In this review, we summarize our current understanding of the biology of these DLBCL subtypes with a special emphasis on novel diagnostic and therapeutic approaches.
\end{abstract}

Keywords: biology, DLBCL, gene expression profiling, malignant lymphoma, molecular pathogenesis

\section{Introduction}

Diffuse large B-cell lymphoma (DLBCL), the most common subtype of malignant lymphoma, is characterized by heterogeneity with respect to clinical presentation, morphology, and molecular pathogenesis [Coiffier, 2001]. The introduction of gene expression profiling revealed the existence of at least three distinct molecular subtypes of DLBCL that differ in the expression of thousands of genes and that seem to arise from B cells at different stages of differentiation [Rosenwald et al. 2003, 2002; Savage et al. 2003; Wright et al. 2003; Alizadeh et al. 2000]. According to their gene expression profiles these subtypes were termed germinal-center B-cell-like (GCB) DLBCL, activated B-cell-like (ABC) DLBCL, and primary mediastinal B-cell lymphoma (PMBL). These subtypes do not only differ with respect to their gene expression profiles, but they depend on different oncogenic pathways and are characterized by significant differences in overall survival following standard treatment [Lenz et al. 2008b, 2008c; Monti et al. 2005; Rosenwald et al. 2002]. Whereas patients with GCB DLBCL respond favorably to a combined approach of the anti-CD20 antibody rituximab and chemotherapy, more than $50 \%$ of $\mathrm{ABC}$ DLBCL patients will succumb to their disease [Lenz et al. 2008b]. This implies that novel therapeutic strategies are critically warranted to improve prognosis of affected patients. However, this can only be achieved by a better understanding of the underlying molecular mechanisms that drive lymphomagenesis.

\section{Molecular pathogenesis of GCB DLBCL}

GCB DLBCLs seem to originate from germinalcenter B-cells (Figure 1) [Rosenwald et al. 2002; Alizadeh et al. 2000]. Accordingly, these lymphomas characteristically express many genes such as $B C L 6$ or $L M O 2$ that are expressed by normal germinal-center B-cells [Alizadeh et al. 2000]. Further evidence for their germinal-center derivation is provided by the fact that GCB DLBCLs frequently show ongoing somatic hypermutation of their variable immunoglobulin heavy chain gene that is mediated by AID, an enzyme that is characteristically expressed at high levels in germinal-center B-cells [Lossos et al. 2000].

Various oncogenic pathways are deregulated in GCB DLBCL that contribute to the molecular pathogenesis of this entity (Figure 1). In approximately $45 \%$ of GCB DLBCL patient samples, a $\mathrm{t}(14 ; 18)$ translocation juxtaposing the BCL2 gene and the $\operatorname{IgH}$ locus is detectable. This leads to constitutive activation of the anti-apoptotic BCL2 protein. Intriguingly, this abnormality is not detectable in ABC DLBCL patient samples [Rosenwald et al. 2002].

Another hallmark of GCB DLBCL biology is the deregulation of the phosphatase and tensin homologue (PTEN)- phosphatidylinositol 3-kinase
Ther Adv Hematol (2011) 2(6) 369-379 DOI: $10.1177 /$ 2040620711419001

(c) The Author(s), 2011. Reprints and permissions: http://www.sagepub.co.uk/ journalsPermissions.nav

Correspondence to: Georg Lenz, MD Department of Hematology, Oncology and Tumor Immunology, Charité -

Universitätsmedizin Berlin, Molecular Cancer Research Center, Augustenburger Platz 1 . 13353 Berlin, Germany georg.lenzacharite.de

Mareike Frick Bernd Dörken Department of Hematology, Oncology and Tumor Immunology, Charité -

Universitätsmedizin Berlin, Germany 


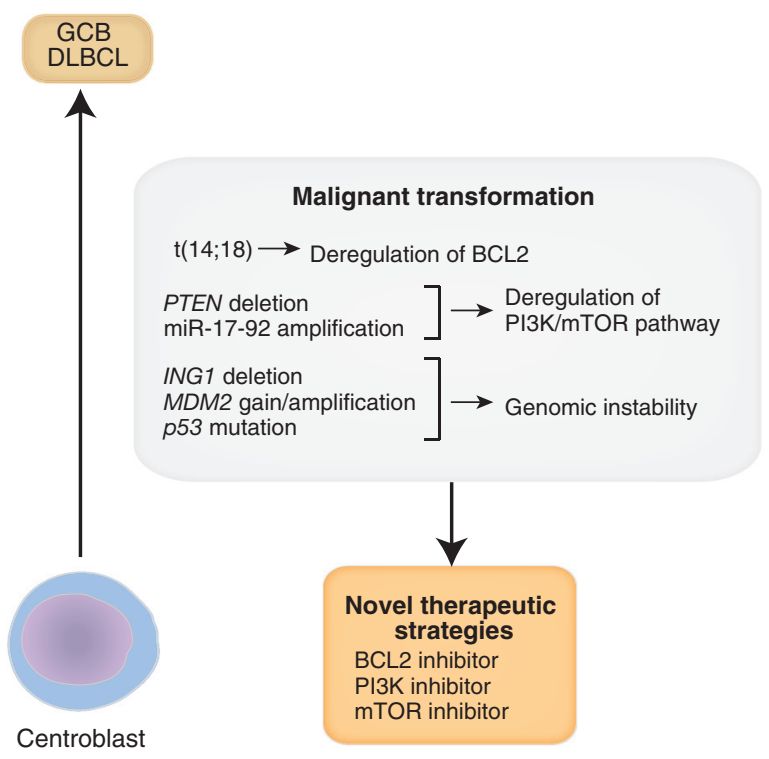

Figure 1. Germinal-center B-cell-like (GCB) diffuse large B-cell lymphoma (DLBCL) derived from normal germinal-center centroblasts. BCL2 is deregulated by $\mathrm{t}(14 ; 18)$ translocations. PTEN deletions and amplification of miR-17-92 lead to deregulation of the PI3K/mTOR pathway. ING1 deletions, p53 mutations, and MDM2 gain/ amplification are associated with genomic instability. Addiction to these signaling pathways conveys vulnerability to specific inhibitors.

(PI3K) signaling pathway (Figure 1) [Lenz et al. 2008c]. The PI3K signaling cascade is initiated with the phosphorylation of phosphatidylinositol 4,5-bisphosphate $\left(\mathrm{PIP}_{2}\right)$ to phosphatidylinositol 3,4,5-trisphosphate $\left(\mathrm{PIP}_{3}\right)$, resulting in cellular processes such as proliferation, cell survival, and cell growth [Chalhoub and Baker, 2009; Salmena et al. 2008]. The conversion of $\mathrm{PIP}_{2}$ into $\mathrm{PIP}_{3}$ is tightly regulated by the opposing activities of the lipid phosphatase PTEN and class I PI3K family members. Whereas the PI3Ks catalyze the conversion from $\mathrm{PIP}_{2}$ to $\mathrm{PIP}_{3}$, PTEN hydrolyzes the 3-phosphate to generate $\mathrm{PIP}_{2}$ [Salmena et al. 2008]. Upon PTEN loss, $\mathrm{PIP}_{3}$ accumulates and AKT and mTOR are activated, further promoting cell survival, proliferation, and growth [Salmena et al. 2008]. Roughly 15\% of GCB DLBCLs harbor heterozygous or homozygous deletions of PTEN. As an alternative mechanism of deregulating PTEN, 15\% of GCB DLBCLs have amplifications of the miR-17-92 locus resulting in PTEN suppression [Lenz et al. 2008c; Xiao et al. 2008]. These findings implicate an important role of the PTEN-PI3K pathway in the biology of GCB DLBCL. Strikingly, these abnormalities are not detectable in either $A B C$ DLBCL or PMBL samples.

GCB DLBCLs are furthermore characterized by gain or amplification of $M D M 2$, a negative regulator of the tumor suppressor p53, as well as deletions of the known tumor suppressor genes TP73 and ING1 (Figure 1) [Lenz et al. 2008c]. Finally, in both GCB DLBCL and PMBL, a locus on the chromosomal arm $2 p$ is recurrently amplified [Lenz et al. 2008c; Bea et al. 2005]. Rel, coding for a subunit of the nuclear factor kappa B $(\mathrm{NF}-\kappa \mathrm{B})$ transcription factor, is among the most upregulated genes within this abnormality. Whereas the NF- $\kappa \mathrm{B}$ signaling pathway is constitutively activated in virtually all $A B C$ DLBCL cases, GCB DLBCLs do not seem to depend on this pathway. Thus, the functional role of this abnormality in the pathogenesis of GCB DLBCL needs to be elucidated.

\section{Molecular pathogenesis of ABC DLBCL}

The gene expression profiles of ABC DLBCL patient samples suggest that this subtype is derived from $B$ cells that are in the process of differentiating into plasma cells (Figure 2) [Rosenwald et al. 2002]. In contrast to GCB DLBCL, most genes characteristically expressed by normal germinal-center B-cells are downregulated in ABC DLBCL. However, these samples exhibit an upregulation of many genes normally expressed in plasma cells [Wright et al. 2003]. Specifically, ABC DLBCLs express XBP-1, a key regulator of the secretory phenotype of plasma cells [Shaffer et al. 2004; Wright et al. 


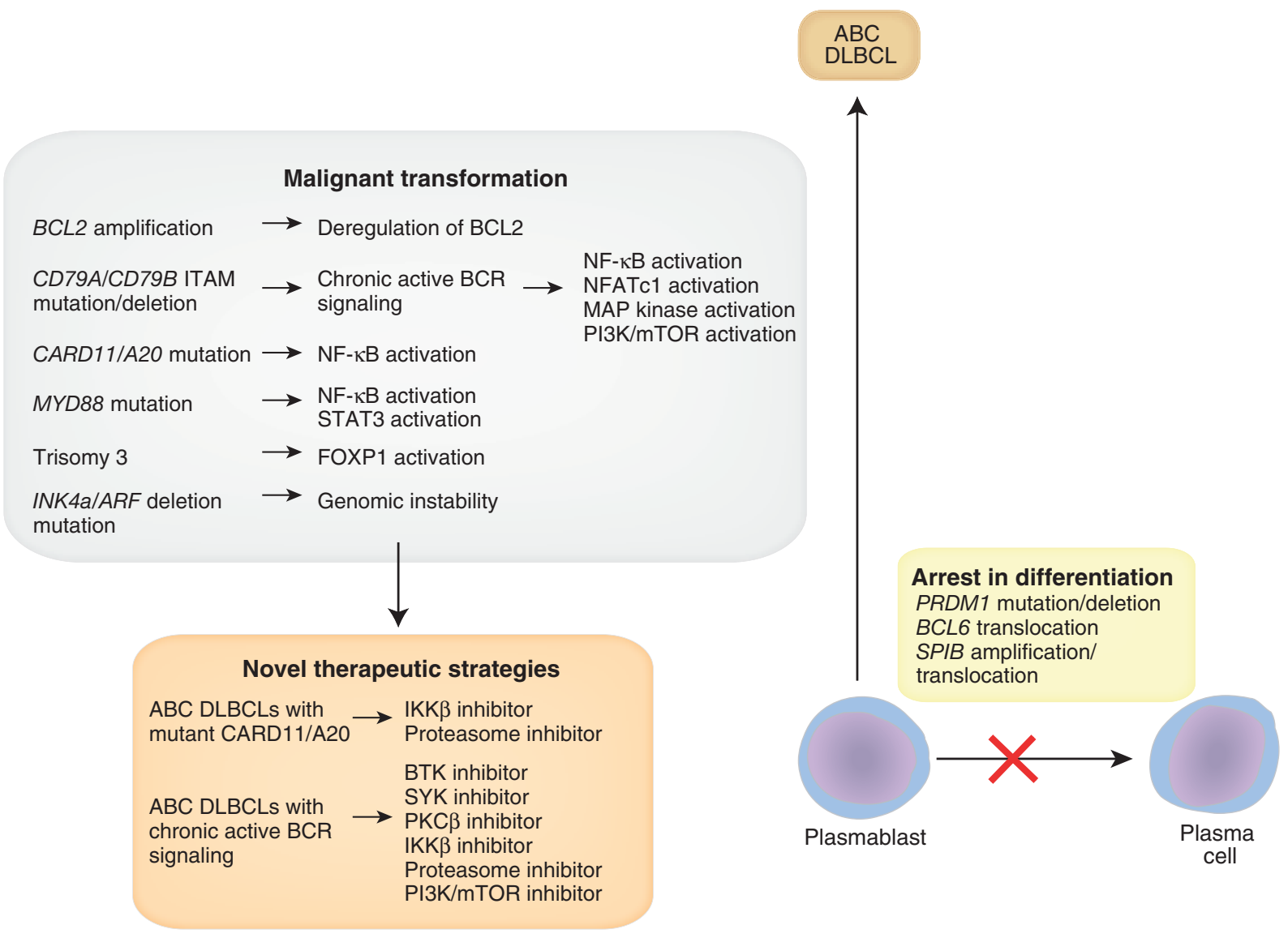

Figure 2. Activated B-cell-like (ABC) diffuse large B-cell lymphomas (DLBCLs) are derived from plasmablasts that are characterized by a block in differentiation. ABC DLBCLs show constitutive activation of the oncogenic nuclear factor kappa $B(N F-\kappa B$ ) signaling pathway as well as deregulation of the anti-apoptotic BCL2 protein. The molecular mechanisms by which NF- $\kappa B$ is activated will determine responsiveness to specific inhibitors.

2003]. However, full plasmacytic differentiation is blocked by different genetic abnormalities (Figure 2). Approximately $25 \%$ of ABC DLBCL patient samples harbor inactivating mutations of PRDM1 which encodes BLIMP1 [Pasqualucci et al. 2006; Tam et al. 2006]. BLIMP1 promotes plasmacytic differentiation by terminating the expression of most mature B-cell differentiation genes [Shaffer et al. 2002]. Recently, overexpression of microRNAs such as let- 7 has been postulated as an alternative mechanism of BLIMP1 deregulation [Nie et al. 2010]. Plasmacytic differentiation is blocked in ABC DLBCL additionally by translocations affecting the PRDM1 repressor BCL6 that are detectable in roughly $25 \%$ of patients [Iqbal et al. 2007; Tunyaplin et al. 2004; Shaffer et al. 2000]. Finally, $26 \%$ of cases show gain/amplification and consecutive overexpression of the ETS family transcription factor SPIB that also represses Blimp-1 expression [Lenz et al. 2008c; Schmidlin et al. 2008]. In summary, the inactivation of PRDM1 by different genetic aberrations provides direct evidence that a block in differentiation is a hallmark of ABC DLCBL biology.

ABC DLBCL is characterized by additional genetic abnormalities that play an important role in its pathogenesis (Figure 2). The INKal $A R F$ tumor suppressor locus is deleted in approximately $30 \%$ of ABC DLBCLs [Lenz et al. 2008c; Tagawa et al. 2005]. Another characteristic abnormality is gain or amplification of $18 \mathrm{q}$ that is associated with overexpression of the anti-apoptotic BCL2 protein [Lenz et al. 2008c; Bea et al. 2005]. In addition, trisomy 3 is frequently detected in ABC DLBCL, but not in other subtypes [Lenz et al. 2008c; Bea et al. 2005]. FOXP1 emerged as a potential target gene of trisomy 3. Previously, FOXP1 has been implicated as an oncogene based on translocations in MALT lymphoma and DLBCL [Lenz et al. 2008c; Haralambieva et al. 2006; 
Streubel et al. 2005]. However, its role in the molecular pathogenesis needs to be validated experimentally.

Another pathogenetic feature of ABC DLBCLs is the constitutive activation of the NF- $\kappa B$ signaling pathway that promotes cell survival and proliferation and inhibits apoptosis (Figure 2) [Davis et al. 2001]. NF- $\kappa \mathrm{B}$ is a family of inducible transcription factors consisting of five members, RelA (p65), RelB, c-Rel, NF-kB1 (p50 and its precursor p105), and NF-kB2 (p52 and its precursor p100) [Jost and Ruland, 2007]. These proteins form different homodimers and heterodimers that are kept inactive by binding to inhibitory proteins of the I $\kappa \mathrm{B}$ family. Activation of NF- $\kappa \mathrm{B}$ through various stimuli leads to phosphorylation and proteosomal degradation of the I $\mathrm{KB}$ proteins by the multiprotein I $\mathrm{KB}$ kinase (IKK) resulting in nuclear translocation of the $\mathrm{NF}-\kappa \mathrm{B}$ subunits and transactivation of their target genes [Jost and Ruland, 2007]. ABC DLBCLs are characterized by constitutive activation of the NF- $\kappa$ B pathway. Inhibition of this pathway using either a dominant active form of I $\mathrm{B} \alpha$ or a specific IKK inhibitor is toxic to ABC but not to GCB DLBCL cell lines indicating the importance of NF- $\mathrm{KB}$ signaling in ABC DLBCL biology [Lam et al. 2005; Davis et al. 2001].

Insights which upstream molecular mechanisms lead to the constitutive activity of NF- $\mathrm{NB}$ in $\mathrm{ABC}$ DLBCL were obtained by a small hairpin RNA (shRNA) screen that revealed toxicity of shRNAs directed against CARD11, BCL10, and MALT1 in ABC DLBCL cell lines [Ngo et al. 2006]. In normal $\mathrm{B}$ and $\mathrm{T}$ cells these three proteins form a transient signaling complex (the CBM complex) following stimulation that activates IKK [Rawlings et al. 2006]. In contrast, in ABC DLBCL the CBM complex is constitutively activated due to different mechanisms. Resequencing revealed that approximately $10 \%$ of $\mathrm{ABC}$ DLBCL patients harbor activating CARD11 mutations leading to NF- $\mathrm{KB}$ pathway activation [Lenz et al. 2008a]. ABC DLBCLs with wildtype CARD11 depend on chronic active B-cell receptor (BCR) signaling. Roughly $20 \%$ of ABC DLBCLs have mutations in the signaling molecules $C D 79 B$ and less frequently in $C D 79 A$. These mutations predominantly affect the ITAM signaling motifs, resulting in reduced activation of LYN, a negative regulator of BCR signaling and increased BCR cell surface expression. These findings implicate chronic
BCR signaling in the biology of ABC DLBCLs with wildtype CARD11 [Davis et al. 2010].

Additional mechanisms for the constitutive activation of $\mathrm{NF}-\kappa \mathrm{B}$ have been reported. Approximately one third of ABC DLBCL patients carry biallelic inactivation of the negative NF- $\mathrm{KB}$ regulator A20 [Compagno et al. 2009; Kato et al. 2009]. Reintroduction of A20 into A20-deficient cell lines resulted in inhibition of $\mathrm{NF}-\kappa \mathrm{B}$ signaling and induced apoptosis and cell growth arrest [Compagno et al. 2009]. Alternatively, a recent study showed that activating mutations affecting the adaptor protein MYD88 that mediates toll and interleukin-1 receptor signaling results in constitutive NF- $\mathrm{NB}$ signaling [Ngo et al. 2011].

\section{Molecular pathogenesis of PMBL}

PMBL has been identified as a distinct entity based on clinical and morphological features. PMBL normally affects young women with a median age of 30-35 years with the mediastinum being the predominant site of lymphoma manifestation. Morphologically PMBL tumors are frequently sclerotic and are characterized by diffuse proliferation of medium-sized to large B-cells with a heterogeneous morphology [van Besien et al. 2001]. However, PMBL cannot be reliably diagnosed solely by morphologic and clinical parameters. A recent gene expression profiling study showed that approximately one quarter of cases diagnosed as PMBL by conventional criteria did not exhibit the characteristic gene expression signature of PMBL and thus represented other molecular DLBCL subtypes with mediastinal involvement [Rosenwald et al. 2003]. Interestingly, PMBLs share many clinical and molecular features with nodular sclerosis classical Hodgkin lymphoma (cHL) [Rosenwald et al. 2003; Savage et al. 2003]. Similar to PMBL, nodular sclerosis cHL often affects young women with a mediastinal mass that is sclerotic in the histological examination. With respect to gene expression profiling, more than one third of the genes overexpressed in PMBL are also highly expressed in cHL cell lines [Rosenwald et al. 2003]. Furthermore, PMBL and nodular sclerosis cHL utilize the same oncogenic pathways such as NF-кB [Rosenwald et al. 2003; Savage et al. 2003]. However, despite these similarities PMBL and nodular sclerosis cHL can reliably be distinguished by histology and gene expression profiling. 
PMBLs are characterized by gain or amplification of the chromosomal band 9p24 [Lenz et al. 2008 c]. Roughly $50 \%$ of PMBL cases harbor this abnormality. One target gene of this amplicon is JAK2, a tyrosine kinase which regulates cytokine signaling through STAT transcription factors. Interestingly, SOCS1, a suppressor of JAK signaling is recurrently deleted in PMBL [Weniger et al. 2006; Mestre et al. 2005]. SOCS1 inhibits JAK2 activity by competing with STAT proteins for the JAK2 binding site [Ritz et al. 2008]. Thus, deletion of SOCS1 contributes to the constitutive activation of the JAK2 pathway implying that JAK2 plays an important role in the molecular pathogenesis of PMBL [Melzner et al. 2006, 2005].

A recent shRNA library screen revealed that additional genes within the 9p24 amplicon might be involved in the biology of PMBL [Rui et al. 2010]. The histone demethylase JMJD2C seems to cooperate with JAK2 to modify the PMBL epigenome and thereby promotes proliferation and survival in PMBL cell lines [Rui et al. 2010]. Thus, inhibition of both JAK2 and JMJD2C might represent a novel therapeutic approach for PMBL. PD-L1 and PD-L2 that are ligands for the PD receptor on T-cells are also upregulated by this amplification in PMBL [Rosenwald et al. 2003]. Engagement of the PD receptor by its ligands inhibits signaling through the T-cell receptor, suggesting that amplification of these genes modulates the interaction between PMBL cells and the surrounding $\mathrm{T}$ cells [Rosenwald et al. 2003]. Finally, PMBLs are characterized by constitutive activation of the NF- $\kappa \mathrm{B}$ signaling pathway [Rosenwald et al. 2003; Savage et al. 2003]. Inhibition of $\mathrm{NF}-\kappa \mathrm{B}$ using a specific IKK inhibitor was toxic to PMBL cell lines, indicating that constitutive activation of this pathway is important for PMBL biology [Lam et al. 2005]. The molecular mechanisms leading to this constitutive activation are poorly understood. In more than $30 \%$ of PMBL cases, the negative NF- $\mathrm{NB}$ regulator $\mathrm{A} 20$ is inactivated [Schmitz et al. 2009].

\section{Clinical implications}

The DLBCL subtypes are characterized by different overall survival rates following standard anthracycline-containing chemotherapy [Monti et al. 2005; Rosenwald et al. 2002]. GCB DLBCL and PMBL patients have relatively favorable 5-year overall survival rates.
In contrast, the majority of ABC DLBCL patients will succumb to their disease. The introduction of the anti-CD20 antibody rituximab significantly improved the outcome for DLBCL patients [Coiffier et al. 2002]. This benefit is observed in both ABC and GCB DLBCL patients. However, GCB DLBCL patients still do significantly better compared with patients diagnosed with ABC DLBCL (Figure 3A) [Lenz et al. 2008b].

Gene expression profiling can provide additional information besides the distinction of $\mathrm{ABC}$ and GCB DLBCL. A multivariate gene expressionbased survival predictor model that is independent of the International Prognostic Index (IPI) can be used to predict survival upfront at diagnosis in DLBCL patients treated with a combination of rituximab and anthracyclinecontaining chemotherapy [Lenz et al. 2008b]. Three gene expression signatures termed 'germinal-center B-cell', 'stromal-1' and 'stromal-2' are associated with survival (Figure $3 \mathrm{~B}$ ). The prognostically favorable 'germinal-center B-cell' signature parallels the distinction of $\mathrm{ABC}$ and GCB DLBCL and consists of genes characteristically expressed in germinal-center B-cells. In contrast, the 'stromal-1' and 'stromal-2' signatures reflect the composition of nonmalignant cells, i.e. the tumor microenvironment [Lenz et al. 2008b]. The favorable 'stromal-1' signature reflects extracellular matrix deposition and histiocytic infiltration. In contrast, the prognostically unfavorable 'stromal-2' signature reflects tumor blood-vessel density. A multivariate model for survival was created from these three gene expression signatures. For every patient biopsy, a survival predictor score was calculated. These scores were used to divide the patients into quartiles of 3-year overall survival rates of $89 \%, 82 \%, 74 \%$, and $48 \%$ and 3-year progression-free survival rates of $84 \%$, $69 \%, 61 \%$, and 33\% (Figure 3B) [Lenz et al. 2008b].

A different approach to utilize changes in gene expression to predict outcome in DLBCL patients was taken by Lossos and colleagues [Lossos et al. 2004]. A predictor of survival measuring only six genes (LMO2, BCL6, FN1, CCND2, SCYA3, and BCL2) was constructed that divided DLBCL patients into low-, medium-, and high-risk groups according to their overall survival [Lossos et al. 2004]. This predictor was recently evaluated and confirmed 
(A)

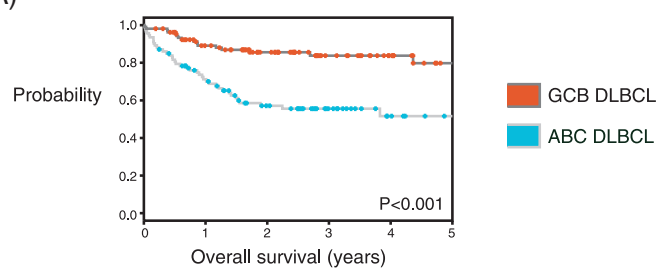

(B)
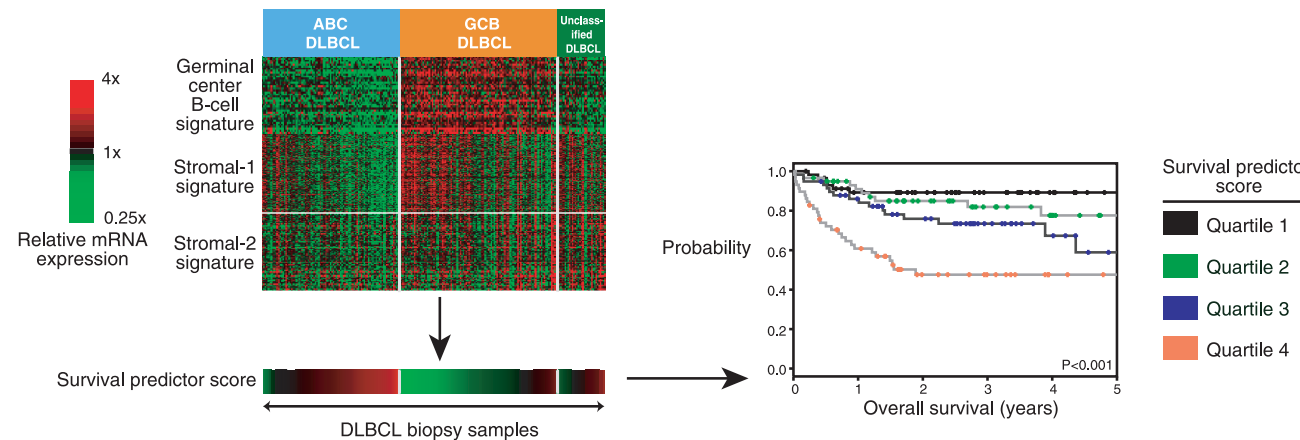

3-year overall survival rates

$89 \%$

$82 \%$

$74 \%$

$48 \%$

Figure 3. (A) Activated B-cell-like (ABC) and germinal-center B-cell-like (GCB) diffuse large B-cell lymphomas (DLBCLs) are characterized by significant differences in overall survival following a combination of rituximab and chemotherapy. (B) A multivariate gene expression-based survival model can be used to predict survival upfront at diagnosis in DLBCL patients treated with a combination of rituximab and chemotherapy. For every patient biopsy, a survival predictor score can be calculated. These scores are used to divide DLBCL patients into quartiles of significantly different 3 -year overall survival rates of $89 \%, 82 \%, 74 \%$, and $48 \%$.

in its predictive value in DLBCL patients using formalin-fixed paraffin-embedded (FFPE) patient material [Malumbres et al. 2008]. Although this approach does not distinguish DLBCL patients into ABC and GCB DLBCL, its application might be helpful for guiding clinical strategies and therefore its implementation could be useful until gene expression profiling techniques are widely available.

In addition to its value in predicting patient outcome, gene expression profiling using DNA microarrays can additionally be applied to distinguish DLBCL from Burkitt's lymphoma (BL). Two recent studies convincingly showed that $\mathrm{BL}$ is characterized by a unique gene expression profile [Dave et al. 2006; Hummel et al. 2006]. Intriguingly, in these analyses a substantial number of cases that were diagnosed as DLBCL by conventional methods showed the gene expression profile of $\mathrm{BL}$, suggesting that they represent BL cases that are difficult to diagnose by current methods. This distinction however is of major clinical importance, as BL requires more intensive treatment strategies.

\section{Future developments}

Despite the recognition and acknowledgement of different molecular DLBCL subtypes more than a decade ago, the translation into clinical routine is only slowly progressing and microarray-based technologies are just emerging in the clinical application. Several efforts to use immunohistochemistry to distinguish the molecular subtypes have been undertaken [Choi et al. 2009; Hans et al. 2004]. A recent report by Meyer and colleagues introduced a novel algorithm (Tally) that seems to be able to distinguish GCB and nonGCB DLBCL subtypes analyzing five immunohistochemical makers (CD10, GCET1, MUM1, FOXP1, and LMO2) [Meyer et al. 2011]. This algorithm showed a high concordance with gene expression profiling and the two identified groups of patients had different overall survival rates. However, this algorithm needs to be confirmed by other groups, especially as previous immunohistochemical approaches to determine the molecular DLBCL subtypes could not always be confirmed in additional analyses. This might be due to the limitations of immunohistochemistry, as staining and scoring procedures are 


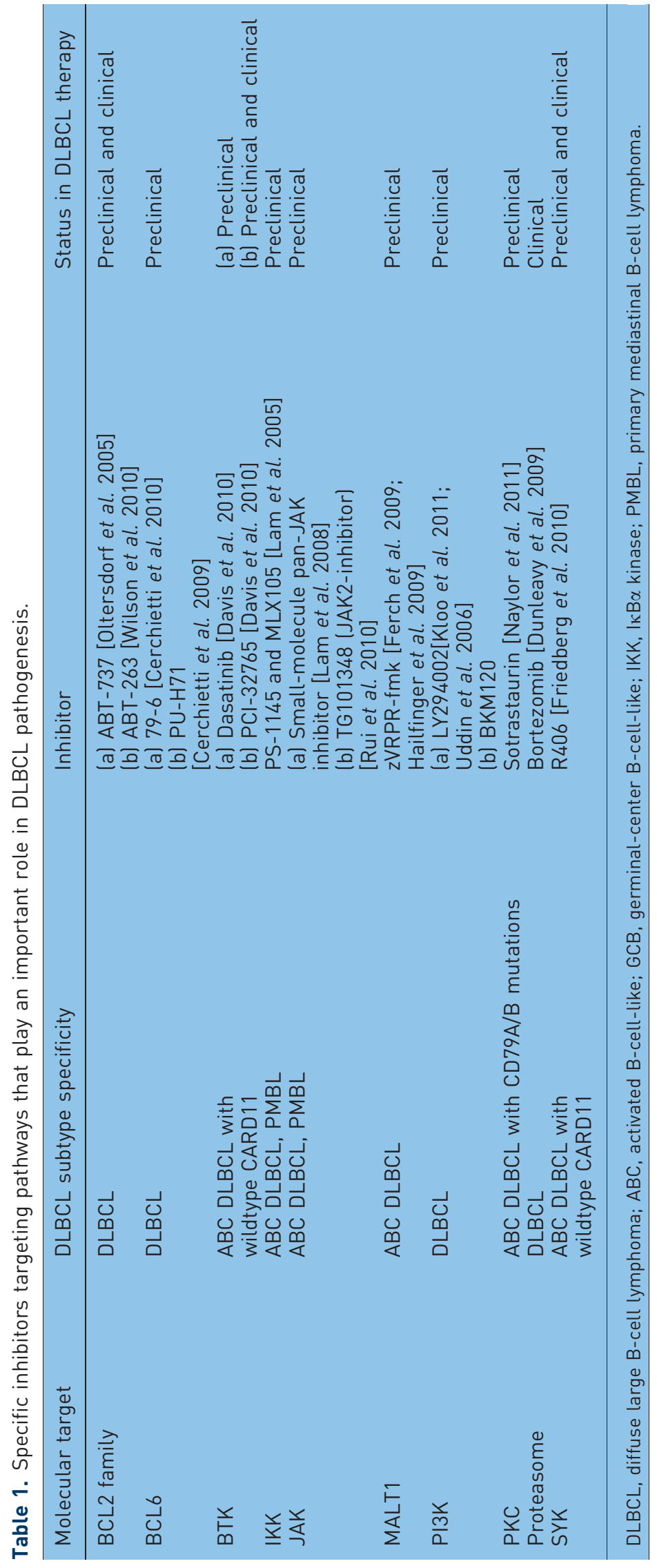


variable even between experienced laboratories as shown by a recent study of the Lunenburg Lymphoma Biomarker Consortium. Therefore, technical variations between laboratories should be harmonized, before these algorithms can be reliably implemented into routine diagnostics [de Jong et al. 2009].

Recently, a quantitative nuclease protection assay was applied on FFPE tissue to distinguish DLBCL patient samples into $A B C$ and GCB DLBCL [Rimsza et al. 2011]. Classification into the molecular subtypes using this assay was compared with results obtained by gene expression profiling applying DNA microarrays. $73 \%$ of GCB DLBCLs and $79 \%$ of ABC DLBCLs were categorized correctly using this assay (confidence cutoff $>0.9$ ) [Rimsza et al. 2011]. As the classification into molecular subtypes will be indispensable in the future for the rational design of clinical studies and the application of new pharmacologic agents, the use of this quantitative nuclease protection assay might hold promise due to its applicability on FFPE patient material. However, gene expression profiling remains the gold standard to distinguish the molecular DLBCL subtypes especially as there has been significant progress in the development of microarray protocols that can be performed on FFPE tissue [Williams et al. 2010].

In the last couple of years significant progress has been made in the development of novel compounds that specifically target oncogenic pathways (summarized in Table 1). Several of these strategies are currently being evaluated in clinical trials. However, these efforts will only be successful if the molecular target or signaling pathway is indeed expressed or utilized by the malignant cells. Thus, it is important to employ our molecular understanding of these diseases and incorporate techniques such as gene expression profiling that can be used to predict if a specific tumor is addicted to a certain oncogenic pathway.

Due to their dependency on $\mathrm{NF}-\kappa \mathrm{B}$ signaling, components of the NF- $\kappa \mathrm{B}$ and/or BCR pathways might represent a promising therapeutic target for ABC DLBCL patients (Table 1). Specific inhibitors targeting PKC and MALT1 showed promising results in preclinical models [Naylor et al. 2011; Ferch et al. 2009; Hailfinger et al. 2009]. It is conceivable that these therapeutic approaches can be translated into the clinic. This was underscored by a recent study in which $\mathrm{ABC}$ and GCB DLBCL patients were treated with a combined approach of chemotherapy and the proteasome inhibitor bortezomib that can inhibit NF- $\kappa \mathrm{B}$ signaling [Dunleavy et al. 2009]. Strikingly, only ABC DLBCL patients showed benefit by the addition of bortezomib. GCB DLBCL might preferentially respond to PI3K or $\mathrm{mTOR}$ inhibitors due to their dependency on the PTEN-PI3K pathway. Finally, PMBL might benefit from the addition of JAK2 inhibitors (Table 1). These efforts will eventually lead to more specific and effective and less-toxic treatment strategies of DLBCL patients.

\section{Funding}

This work was supported by research grants to GL from the German Research Foundation (DFG), the Deutsche Krebshilfe, the Berliner Krebsgesellschaft e.V., and the Else KrönerFresenius-Stiftung.

\section{Conflict of interest statement}

The authors declare no conflicts of interest in preparing this article.

\section{References}

Alizadeh, A.A., Eisen, M.B., Davis, R.E., Ma, C., Lossos, I.S., Rosenwald, A. et al. (2000) Distinct types of diffuse large B-cell lymphoma identified by gene expression profiling. Nature 403: 503-511.

Bea, S., Zettl, A., Wright, G., Salaverria, I., Jehn, P., Moreno, V. et al. (2005) Diffuse large B-cell lymphoma subgroups have distinct genetic profiles that influence tumor biology and improve gene-expression-based survival prediction. Blood 106: 3183-3190.

Cerchietti, L.C., Ghetu, A.F., Zhu, X., Da Silva, G.F., Zhong, S., Matthews, M. et al. (2010) A small-molecule inhibitor of BCL6 kills DLBCL cells in vitro and in vivo. Cancer Cell 17: 400-411.

Cerchietti, L.C., Lopes, E.C., Yang, S.N., Hatzi, K., Bunting, K.L., Tsikitas, L.A. et al. (2009) A purine scaffold Hsp90 inhibitor destabilizes BCL-6 and has specific antitumor activity in BCL-6-dependent B cell lymphomas. Nat Med 15: 1369-1376.

Chalhoub, N. and Baker, S.J. (2009) PTEN and the PI3-kinase pathway in cancer. Annu Rev Pathol 4: $127-150$.

Choi, W.W., Weisenburger, D.D., Greiner, T.C., Piris, M.A., Banham, A.H., Delabie, J. et al. (2009) A new immunostain algorithm classifies diffuse large B-cell lymphoma into molecular subtypes with high accuracy. Clin Cancer Res 15: 5494-5502.

Coiffier, B. (2001) Diffuse large cell lymphoma. Curr Opin Oncol 13: 325-334. 
Coiffier, B., Lepage, E., Briere, J., Herbrecht, R., Tilly, H., Bouabdallah, R. et al. (2002) CHOP chemotherapy plus rituximab compared with CHOP alone in elderly patients with diffuse large-B-cell lymphoma. $N$ Engl f Med 346: 235-242.

Compagno, M., Lim, W.K., Grunn, A., Nandula, S.V., Brahmachary, M., Shen, Q. et al. (2009) Mutations of multiple genes cause deregulation of NF-kappaB in diffuse large B-cell lymphoma. Nature 459: 717-721.

Dave, S.S., Fu, K., Wright, G.W., Lam, L.T., Kluin, P., Boerma, E.J. et al. (2006) Molecular diagnosis of Burkitt's lymphoma. N Engl F Med 354: 2431-2442.

Davis, R.E., Brown, K.D., Siebenlist, U. and Staudt, L.M. (2001) Constitutive nuclear factor kappaB activity is required for survival of activated B cell-like diffuse large B cell lymphoma cells. $\mathcal{F}$ Exp Med 194: 1861-1874.

Davis, R.E., Ngo, V.N., Lenz, G., Tolar, P., Young, R.M., Romesser, P.B. et al. (2010) Chronic active Bcell-receptor signalling in diffuse large B-cell lymphoma. Nature 463: 88-92.

de Jong, D., Xie, W., Rosenwald, A., Chhanabhai, M., Gaulard, P., Klapper, W. et al. (2009)

Immunohistochemical prognostic markers in diffuse large B-cell lymphoma: validation of tissue microarray as a prerequisite for broad clinical applications (a study from the Lunenburg Lymphoma Biomarker Consortium). f Clin Pathol 62: 128-138.

Dunleavy, K., Pittaluga, S., Czuczman, M.S., Dave, S.S., Wright, G., Grant, N. et al. (2009) Differential efficacy of bortezomib plus chemotherapy within molecular subtypes of diffuse large B-cell lymphoma. Blood 113: 6069-6076.

Ferch, U., Kloo, B., Gewies, A., Pfander, V., Duwel, M., Peschel, C. et al. (2009) Inhibition of MALT1 protease activity is selectively toxic for activated B celllike diffuse large B cell lymphoma cells. 7 Exp Med 206: 2313-2320.

Friedberg, J.W., Sharman, J., Sweetenham, J., Johnston, P.B., Vose, J.M., Lacasce, A. et al. (2010) Inhibition of Syk with fostamatinib disodium has significant clinical activity in non-Hodgkin lymphoma and chronic lymphocytic leukemia. Blood 115: 2578-2585.

Hailfinger, S., Lenz, G., Ngo, V., Posvitz-Fejfar, A., Rebeaud, F., Guzzardi, M. et al. (2009) Essential role of MALT1 protease activity in activated B cell-like diffuse large B-cell lymphoma. Proc Natl Acad Sci U S A 106: 19946-19951.

Hans, C.P., Weisenburger, D.D., Greiner, T.C., Gascoyne, R.D., Delabie, J., Ott, G. et al. (2004) Confirmation of the molecular classification of diffuse large B-cell lymphoma by immunohistochemistry using a tissue microarray. Blood 103: 275-282.

Haralambieva, E., Adam, P., Ventura, R., Katzenberger, T., Kalla, J., Holler, S. et al. (2006) Genetic rearrangement of FOXP1 is predominantly detected in a subset of diffuse large B-cell lymphomas with extranodal presentation. Leukemia 20: 1300-1303.

Hummel, M., Bentink, S., Berger, H., Klapper, W., Wessendorf, S., Barth, T.F. et al. (2006) A biologic definition of Burkitt's lymphoma from transcriptional and genomic profiling. $N$ Engl F Med 354: 2419-2430.

Iqbal, J., Greiner, T.C., Patel, K., Dave, B.J., Smith, L., Ji, J. et al. (2007) Distinctive patterns of BCL6 molecular alterations and their functional consequences in different subgroups of diffuse large B-cell lymphoma. Leukemia 21: 2332-2343.

Jost, P.J. and Ruland, J. (2007) Aberrant NF-kappaB signaling in lymphoma: mechanisms, consequences, and therapeutic implications. Blood 109: 2700-2707.

Kato, M., Sanada, M., Kato, I., Sato, Y., Takita, J., Takeuchi, K. et al. (2009) Frequent inactivation of A20 in B-cell lymphomas. Nature 459: 712-716.

Kloo, B., Nagel, D., Pfeifer, M., Grau, M., Duwel, M., Vincendeau, M. et al. (2011) Critical role of PI3K signaling for NF-kappaB-dependent survival in a subset of activated B-cell-like diffuse large B-cell lymphoma cells. Proc Natl Acad Sci U S A 108: 272-277.

Lam, L.T., Davis, R.E., Wright, G., Rosenwald, A., Hurt, E.M.,X.,Y. et al. (2005) Small molecule inhibitors of IkB-kinase are selectively toxic for subgroups of diffuse large $\mathrm{B}$ cell lymphoma defined by gene expression profiling. Clin Cancer Res 11: 28-40.

Lam, L.T., Wright, G., Davis, R.E., Lenz, G., Farinha, P., Dang, L. et al. (2008) Cooperative signaling through the signal transducer and activator of transcription 3 and nuclear factor-\{kappa\}B pathways in subtypes of diffuse large B-cell lymphoma. Blood 111: 3701-3713

Lenz, G., Davis, R.E., Ngo, V.N., Lam, L., George, T.C., Wright, G.W. et al. (2008a) Oncogenic CARD11 mutations in human diffuse large B cell lymphoma. Science 319: 1676-1679.

Lenz, G., Wright, G., Dave, S.S., Xiao, W., Powell, J., Zhao, H. et al. (2008b) Stromal gene signatures in large-B-cell lymphomas. $N$ Engl f Med 359: 2313-2323.

Lenz, G., Wright, G.W., Emre, N.C., Kohlhammer, H., Dave, S.S., Davis, R.E. et al. (2008c) Molecular subtypes of diffuse large B-cell lymphoma arise by distinct genetic pathways. Proc Natl Acad Sci US A 105: 13520-13525.

Lossos, I.S., Alizadeh, A.A., Eisen, M.B., Chan, W.C., Brown, P.O., Botstein, D. et al. (2000) Ongoing immunoglobulin somatic mutation in germinal center $\mathrm{B}$ cell-like but not in activated B cell-like diffuse large cell lymphomas. Proc Natl Acad Sci US A 97: 10209-10213.

Lossos, I.S., Czerwinski, D.K., Alizadeh, A.A., Wechser, M.A., Tibshirani, R., Botstein, D. et al. (2004) Prediction of survival in diffuse large-B-cell lymphoma based on the expression of six genes. $N$ Engl f Med 350: 1828-1837. 
Malumbres, R., Chen, J., Tibshirani, R., Johnson, N.A., Sehn, L.H., Natkunam, Y. et al. (2008) Paraffinbased 6-gene model predicts outcome in diffuse large B-cell lymphoma patients treated with R-CHOP. Blood 111: 5509-5514.

Melzner, I., Bucur, A.J., Bruderlein, S., Dorsch, K., Hasel, C., Barth, T.F. et al. (2005) Biallelic mutation of SOCS-1 impairs JAK2 degradation and sustains phospho-JAK2 action in the MedB-1 mediastinal lymphoma line. Blood 105: 2535-2542.

Melzner, I., Weniger, M.A., Bucur, A.J., Bruderlein, S., Dorsch, K., Hasel, C. et al. (2006) Biallelic deletion within $16 \mathrm{p} 13.13$ including SOCS-1 in Karpas1106P mediastinal B-cell lymphoma line is associated with delayed degradation of JAK 2 protein. Int $\mathcal{F}$ Cancer 118: 1941-1944.

Mestre, C., Rubio-Moscardo, F., Rosenwald, A., Climent, J., Dyer, M.J., Staudt, L. et al. (2005) Homozygous deletion of SOCS1 in primary mediastinal B-cell lymphoma detected by CGH to BAC microarrays. Leukemia 19: 1082-1084.

Meyer, P.N., Fu, K., Greiner, T.C., Smith, L.M., Delabie, J., Gascoyne, R.D. et al. (2011)

Immunohistochemical methods for predicting cell of origin and survival in patients with diffuse large B-cell lymphoma treated with rituximab. $\mathcal{F}$ Clin Oncol 29: 200-207.

Monti, S., Savage, K.J., Kutok, J.L., Feuerhake, F., Kurtin, P., Mihm, M. et al. (2005) Molecular profiling of diffuse large B-cell lymphoma identifies robust subtypes including one characterized by host inflammatory response. Blood 105: 1851-1861.

Naylor, T.L., Tang, H., Ratsch, B.A., Enns, A., Loo, A., Chen, L. et al. (2011) Protein kinase C inhibitor Sotrastaurin selectively inhibits the growth of CD79mutant diffuse large B-cell lymphomas. Cancer Res, in press.

Ngo, V.N., Davis, R.E., Lamy, L., Yu, X., Zhao, H., Lenz, G. et al. (2006) A loss-of-function RNA interference screen for molecular targets in cancer. Nature 441: 106-110.

Ngo, V.N., Young, R.M., Schmitz, R., Jhavar, S., Xiao, W., Lim, K.H. et al. (2011) Oncogenically active MYD88 mutations in human lymphoma. Nature 470: 115-119.

Nie, K., Zhang, T., Allawi, H., Gomez, M., Liu, Y., Chadburn, A. et al. (2010) Epigenetic down-regulation of the tumor suppressor gene PRDM1/Blimp-1 in diffuse large B cell lymphomas: a potential role of the microRNA let-7. Am f Pathol 177: 1470-1479.

Oltersdorf, T., Elmore, S.W., Shoemaker, A.R., Armstrong, R.C., Augeri, D.J., Belli, B.A. et al. (2005) An inhibitor of Bcl-2 family proteins induces regression of solid tumours. Nature 435: 677-681.

Pasqualucci, L., Compagno, M., Houldsworth, J., Monti, S., Grunn, A., Nandula, S.V. et al. (2006) Inactivation of the PRDM1/BLIMP1 gene in diffuse large B cell lymphoma. J Exp Med 203: 311-317.
Rawlings, D.J., Sommer, K. and Moreno-Garcia, M.E. (2006) The CARMA1 signalosome links the signalling machinery of adaptive and innate immunity in lymphocytes. Nat Rev Immunol 6: 799-812.

Rimsza, L.M., Wright, G.W., Schwartz, M., Chan, W., Jaffe, E., Gascoyne, R.D. et al. (2011) Classification of diffuse large B cell lymphoma into germinal center and activated B cell subtypes using a nuclease protection assay on paraffin embedded tissues. Clin Cancer Res, in press.

Ritz, O., Guiter, C., Dorsch, K., Dusanter-Fourt, I., Wegener, S., Jouault, H. et al. (2008) STAT6 activity is regulated by SOCS-1 and modulates BCL-XL expression in primary mediastinal B-cell lymphoma. Leukemia 22: 2106-2110.

Rosenwald, A., Wright, G., Chan, W.C., Connors, J.M., Campo, E., Fisher, R.I. et al. (2002) The use of molecular profiling to predict survival after chemotherapy for diffuse large-B-cell lymphoma. $N \mathrm{Engl} \mathcal{F}$ Med 346: 1937-1947.

Rosenwald, A., Wright, G., Leroy, K., Yu, X., Gaulard, P., Gascoyne, R.D. et al. (2003) Molecular diagnosis of primary mediastinal B cell lymphoma identifies a clinically favorable subgroup of diffuse large B cell lymphoma related to Hodgkin lymphoma. f Exp Med 198: 851-862.

Rui, L., Emre, N.C., Kruhlak, M.J., Chung, H.J., Steidl, C., Slack, G. et al. (2010) Cooperative epigenetic modulation by cancer amplicon genes. Cancer Cell 18: 590-605.

Salmena, L., Carracedo, A. and Pandolfi, P.P. (2008) Tenets of PTEN tumor suppression. Cell 133: 403-414.

Savage, K.J., Monti, S., Kutok, J.L., Cattoretti, G., Neuberg, D., De Leval, L. et al. (2003) The molecular signature of mediastinal large B-cell lymphoma differs from that of other diffuse large B-cell lymphomas and shares features with classical Hodgkin lymphoma. Blood 102: 3871-3879.

Schmidlin, H., Diehl, S.A., Nagasawa, M., Scheeren, F.A., Schotte, R., Uittenbogaart, C.H. et al. (2008) Spi-B inhibits human plasma cell differentiation by repressing BLIMP1 and XBP-1 expression. Blood 112: $1804-1812$.

Schmitz, R., Hansmann, M.L., Bohle, V., MartinSubero, J.I., Hartmann, S., Mechtersheimer, G. et al. (2009) TNFAIP3 (A20) is a tumor suppressor gene in Hodgkin lymphoma and primary mediastinal B cell lymphoma. J Exp Med 206: 981-989.

Shaffer, A.L., Lin, K.I., Kuo, T.C., Yu, X., Hurt, E.M., Rosenwald, A. et al. (2002) Blimp-1 orchestrates plasma cell differentiation by extinguishing the mature B cell gene expression program. Immunity 17: 51-62.

Shaffer, A.L., Shapiro-Shelef, M., Iwakoshi, N.N., Lee, A.-H., Qian, S.-B., Zhao, H. et al. (2004) XBP1, downstream of Blimp-1, expands the secretory apparatus and other organelles, and increases protein 
synthesis in plasma cell differentiation. Immunity 21: 81-93.

Shaffer, A.L., Yu, X., He, Y., Boldrick, J., Chan, E.P. and Staudt, L.M. (2000) BCL-6 represses genes that function in lymphocyte differentiation, inflammation, and cell cycle control. Immunity 13: 199-212.

Streubel, B., Vinatzer, U., Lamprecht, A., Raderer, M. and Chott, A. (2005) $\mathrm{T}(3 ; 14)(\mathrm{p} 14.1 ; \mathrm{q} 32)$ involving IGH and FOXP1 is a novel recurrent chromosomal aberration in MALT lymphoma. Leukemia 19: $652-658$

Tagawa, H., Suguro, M., Tsuzuki, S., Matsuo, K., Karnan, S., Ohshima, K. et al. (2005) Comparison of genome profiles for identification of distinct subgroups of diffuse large B-cell lymphoma. Blood 106: 1770-1777.

Tam, W., Gomez, M., Chadburn, A., Lee, J.W., Chan, W.C. and Knowles, D.M. (2006) Mutational analysis of PRDM1 indicates a tumor-suppressor role in diffuse large B-cell lymphomas. Blood 107: 4090-4100.

Tunyaplin, C., Shaffer, A.L., Angelin-Duclos, C.D., Yu, X., Staudt, L.M. and Calame, K.L. (2004) Direct repression of prdm 1 by Bcl-6 inhibits plasmacytic differentiation. F Immunol 173: 1158-1165.

Uddin, S., Hussain, A.R., Siraj, A.K., Manogaran, P.S., Al-Jomah, N.A., Moorji, A. et al. (2006) Role of phosphatidylinositol 3'-kinase/AKT pathway in diffuse large B-cell lymphoma survival. Blood 108: 4178-4186.

van Besien, K., Kelta, M. and Bahaguna, P. (2001) Primary mediastinal B-cell lymphoma: a review of pathology and management. $\mathcal{F}$ Clin Oncol 19: 1855-1864.

Weniger, M.A., Melzner, I., Menz, C.K., Wegener, S., Bucur, A.J., Dorsch, K. et al. (2006) Mutations of the tumor suppressor gene SOCS-1 in classical Hodgkin lymphoma are frequent and associated with nuclear phospho-STAT5 accumulation. Oncogene 25: 2679-2684

Williams, P.M., Li, R., Johnson, N.A., Wright, G., Heath, J.D. and Gascoyne, R.D. (2010) A novel method of amplification of FFPET-derived RNA enables accurate disease classification with microarrays. 7 Mol Diagn 12: 680-686.

Wilson, W.H., O'Connor, O.A., Czuczman, M.S., LaCasce, A.S., Gerecitano, J.F., Leonard, J.P. et al. (2010) Navitoclax, a targeted high-affinity inhibitor of BCL-2, in lymphoid malignancies: a phase 1 doseescalation study of safety, pharmacokinetics, pharmacodynamics, and antitumour activity. Lancet Oncol 11: 1149-1159.

Wright, G., Tan, B., Rosenwald, A., Hurt, E.H., Wiestner, A. and Staudt, L.M. (2003) A gene expression-based method to diagnose clinically distinct subgroups of diffuse large B cell lymphoma. Proc Natl Acad Sci U S A 100: 9991-9996.

Xiao, C., Srinivasan, L., Calado, D.P., Patterson, H.C., Zhang, B., Wang, J. et al. (2008)

Lymphoproliferative disease and autoimmunity in mice with increased miR-17-92 expression in lymphocytes. Nat Immunol 9: 405-414.
Visit SAGE journals online http://tah.sagepub.com

(9SAGEJOURNALS 